\title{
ON A RESULT OF PEETRE ABOUT INTERPOLATION OF OPERATOR SPACES
}

\author{
Fernando Cobos* and Teresa Signes** \\ Dedicated to Professor Jaak Peetre with affection \\ and deep admiration for his useful and creative work \\ during the last 40 years
}

Abstract

We establish interpolation formulæ for operator spaces that are components of a given quasi-normed operator ideal. Sometimes we assume that one of the couples involved is quasi-linearizable, some other times we assume injectivity or surjectivity in the ideal. We also show the necessity of these suppositions.

\section{Introduction}

Let $\bar{B}=\left(B_{0}, B_{1}\right)$ be a Banach couple. The Peetre $K$-functional is defined by

$$
K(t, b)=\inf \left\{\left\|b_{0}\right\|_{B_{0}}+t\left\|b_{1}\right\|_{B_{1}}: b=b_{0}+b_{1}, b_{i} \in B_{i}\right\}
$$

where $t>0$ and $b \in B_{0}+B_{1}$. This function plays a major role in Interpolation Theory (see e.g. [15], [1] , [21]); it can be computed explicitly in many concrete cases, and sometimes it coincides with well-known objects in Analysis. For example, in the case of the couple of Lebesgue spaces $\left(L_{1}, L_{\infty}\right)$, one can prove that

$$
K(t, f)=\int_{0}^{t} f^{*}(s) d s
$$

where $f^{*}$ is the non-increasing rearrangement of $f$ on $(0, \infty)$. For the couple $\left(C^{0}, C^{1}\right)$, formed by the space $C^{0}$ of bounded uniformly continuous functions on the real line and the space $C^{1}$ of functions with

1991 Mathematics Subject Classification. 46B70, 47D50.

* Supported in part by DGES (PB97-0254).

** Supported by grant FP-95 of Ministerio de Educación y Ciencia and by DGES (PB97-0254). 
derivatives in $C^{0}$, it turns out that

$$
K(t, f) \approx w(t, f),
$$

that is to say, $K(t, f)$ is equivalent to the modulus of continuity $w(t, f)$ of $f$.

A Banach couple $\bar{B}=\left(B_{0}, B_{1}\right)$ is said to be quasi-linearizable if for any $b \in B_{0}+B_{1}$ one can get in a linear way an optimum decomposition of $b$ for the $K$-functional. We give the precise definition and examples in Section 2. This notion was introduced by Peetre in $[\mathbf{1 6}]$ and $[\mathbf{1 7}]$. It is rather restrictive, but many interesting couples satisfy it (see [21]). Moreover, the idea of quasi-linearization is important for the computation of the $K$-functional in other special instances (see, for example, $[\mathbf{1 2}])$.

Peetre proved in [16] that if $\bar{B}$ is quasi-linearizable then for any Banach space $A$ and any positive measurable function $f=f(t)$, the inclusion

$$
\mathcal{L}\left(A, \bar{B}_{f, \infty ; K}\right) \hookrightarrow\left(\mathcal{L}\left(A, B_{0}\right), \mathcal{L}\left(A, B_{1}\right)\right)_{f, \infty ; K}
$$

is valid, with embedding constant depending only on $\bar{B}$. Here the space $\bar{B}_{f, \infty ; K}$ is defined similarly to the real interpolation space $\bar{B}_{\theta, \infty}$ realized as a $K$-space, but replacing the function $t^{\theta}$ by a more general function $f(t)$ (see Section 2 for more details).

The interest of formula (1.1) is due to its being a converse inclusion to the one given by the interpolation property of the real method, namely

$$
\left(\mathcal{L}\left(A, B_{0}\right), \mathcal{L}\left(A, B_{1}\right)\right)_{\theta, 1} \hookrightarrow \mathcal{L}\left(A, \bar{B}_{\theta, 1}\right) .
$$

It turns out that quasi-linearizable couples $\bar{B}$ can be characterized as those for which (1.1) holds (see [16]).

The aim of this paper is to continue the research on embedding (1.1) in several directions. After reviewing some basic notions in Section 2, we show in Section 3 that (1.1) remains valid if we replace the class of bounded linear operators by any quasi-normed operator ideal $\mathcal{J}$. It is also possible to substitute $\bar{B}_{f, \infty ; K}$ by any intermediate space $B$ with respect to $\bar{B}$. In other words, it holds

$$
\mathcal{J}(A, B) \hookrightarrow\left(\mathcal{J}\left(A, B_{0}\right), \mathcal{J}\left(A, B_{1}\right)\right)_{\psi, \infty ; K} .
$$

Here $\psi=\psi(t)$ is a function associated in a natural way to $B$ and $\bar{B}$. We study then the "dual" situation in which the couple $\bar{B}$ is in the front, and we prove that

$$
\mathcal{J}(B, A) \hookrightarrow\left(\mathcal{J}\left(B_{0}, A\right), \mathcal{J}\left(B_{1}, A\right)\right)_{\rho^{*}, \infty ; K}
$$


where $\rho=\rho(t)$ is another function naturally associated to $B$ and $\bar{B}$ and $\rho^{*}(t)=1 / \rho\left(t^{-1}\right)$. Moreover, the validity of (1.3) implies the quasilinearizability of $\bar{B}$. Other embeddings that arise interpolating by the $J$-method are established in Section 3 as well.

In Section 4 we combine the previous results with some arguments of bilinear type to identify some interpolation spaces between spaces of bounded operators, spaces of nuclear operators and spaces of tensor products. Previous results in this direction are due to Kouba [11] and to Ovchinnikov [13] and [14]. We also establish some interpolation formulæ for approximation numbers and some embeddings for operator ideals defined by approximation numbers.

Finally, in Section 5, we return to the $J$-formulæ, and we show that it is possible to dispense with the quasi-linearizability of $\bar{B}$ provided the operator ideal $\mathcal{J}$ is injective or surjective, and that these conditions on $\mathcal{J}$ are essential for the results.

\section{Preliminaries}

Let $\bar{B}=\left(B_{0}, B_{1}\right)$ be a Banach couple, that is, $B_{0}$ and $B_{1}$ are Banach spaces continuously embedded in some Hausdorff topological vector space. We equip $B_{0}+B_{1}$ [respectively $B_{0} \cap B_{1}$ ] with the norm $K(1, \cdot)$ [respectively $J(1, \cdot)$ ] where for $t>0$

$$
K(t, \cdot)=K\left(t, \cdot ; B_{0}, B_{1}\right) \text { and } J(t, \cdot)=J\left(t, \cdot ; B_{0}, B_{1}\right)
$$

are the Peetre functionals, defined by

$$
K(t, b)=\inf \left\{\left\|b_{0}\right\|_{B_{0}}+t\left\|b_{1}\right\|_{B_{1}}: b=b_{0}+b_{1}, b_{i} \in B_{i}\right\}
$$

and

$$
J(t, b)=\max \left\{\|b\|_{B_{0}}, t\|b\|_{B_{1}}\right\} .
$$

A Banach couple $\bar{B}=\left(B_{0}, B_{1}\right)$ is said to be quasi-linearizable if there exist two families of operators $V_{j}(t) \in \mathcal{L}\left(B_{0}+B_{1}, B_{j}\right), j=0,1,0<t<$ $\infty$ and a constant $k$ such that

$$
\begin{gathered}
\left.V_{0}(t)+V_{1}(t)=I \text { (identity mapping in } B_{0}+B_{1}\right), \\
\left\|V_{0}(t) b\right\|_{B_{0}} \leq k t^{j}\|b\|_{B_{j}}, \quad b \in B_{j} \quad(j=0,1), \\
\left\|V_{1}(t) b\right\|_{B_{1}} \leq k t^{j-1}\|b\|_{B_{j}}, \quad b \in B_{j} \quad(j=0,1),
\end{gathered}
$$

(see $[\mathbf{1 6}]$ or $[\mathbf{1 7}])$.

For instance, the couples $\left(L_{p}\left(w_{0}\right), L_{p}\left(w_{1}\right)\right)$ of weighted $L_{p}$-spaces, or $\left(L_{p}\left(\mathbb{R}^{n}\right), W_{p}\left(\mathbb{R}^{n}\right)\right)$ satisfy this condition. More generally, if $A$ is a Banach space and $D(\Lambda)$ is the domain of the infinitesimal generator 
of a strongly continuous semi-group of operators in $A$, then the couple $(A, D(\Lambda))$ is quasi-linearizable. Another example is the couple of Besov spaces $\left(B_{p, q}^{s_{0}}\left(\mathbb{R}^{n}\right), B_{p, q}^{s_{1}}\left(\mathbb{R}^{n}\right)\right)$. On the other hand, if $p_{0} \neq p_{1}$, the couple $\left(L_{p_{0}}, L_{p_{1}}\right)$ is not quasi-linearizable. See [16] and [21] for more details.

We say that a Banach space $B$ is intermediate with respect to the couple $\bar{B}=\left(B_{0}, B_{1}\right)$ if $B_{0} \cap B_{1} \hookrightarrow B \hookrightarrow B_{0}+B_{1}$, with continuous inclusions. The position of $B$ with respect to $\bar{B}$ can be described by the functions

$$
\psi(t)=\psi(t, B ; \bar{B})=\sup \left\{K(t, b):\|b\|_{B}=1\right\}
$$

and

$$
\rho(t)=\rho(t, B ; \bar{B})=\inf \left\{J(t, b): b \in B_{0} \cap B_{1},\|b\|_{B}=1\right\} .
$$

These functions have been introduced by Cobos, Cwikel and Matos in [2] and they are variants of functions studied by Dmitriev [5] and Pustylnik $[\mathbf{2 0}]$.

We have $0<\rho(t), \psi(t)<\infty$ for all $t>0$; the functions $\psi(t)$ and $\rho(t)$ being non-decreasing, while $\psi(t) / t, \rho(t) / t$ are non-increasing. As an example, let $B$ be a rearrangement invariant space on a $\sigma$-finite measure space $(\Omega, \mu)$ and let $\varphi_{B}$ be its fundamental function, i.e. $\varphi_{B}(t)=\left\|\chi_{E}\right\|_{B}$ where $E \subseteq \Omega$ with $\mu(E)=t$. Then $B$ is an intermediate space with respect to the couple $\left(L_{1}, L_{\infty}\right)$ and

$$
\psi\left(t, B ; L_{1}, L_{\infty}\right)=\rho\left(t, B ; L_{1}, L_{\infty}\right)=t / \varphi_{B}(t) \quad(\text { see }[\mathbf{2 0}]) .
$$

A class $\mathcal{J}$ of bounded linear operators between Banach spaces is said to be an operator ideal if each component $\mathcal{J} \cap \mathcal{L}(A, B)=\mathcal{J}(A, B)$ is a linear subspace of $\mathcal{L}(A, B)$ that contains the finite rank operators and satisfies that $S T R \in \mathcal{J}(E, F)$ whenever $R \in \mathcal{L}(E, A), T \in \mathcal{J}(A, B)$ and $S \in \mathcal{L}(B, F)$.

A non-negative function $\tau: \mathcal{J} \longrightarrow[0, \infty)$ is called a quasi-norm on $\mathcal{J}$ if $\tau$ has the following properties:
(2.6) $\tau(h \otimes b)=\|h\|_{A^{*}}\|b\|_{B}$
for $h \in A^{*}$ and $b \in B$.
(2.7) $\tau(S+T) \leq c(\tau(S)+\tau(T))$
for $S, T \in \mathcal{J}(A, B)$,
where $c=c_{\tau} \geq 1$ is a constant.
(2.8) $\tau(S T R) \leq\|S\|_{B, F} \tau(T)\|R\|_{E, A}$
for $R \in \mathcal{L}(E, A)$,
$T \in \mathcal{J}(A, B)$ and $S \in \mathcal{L}(B, F)$. 
It follows from (2.8) that $\tau(\lambda T)=|\lambda| \tau(T)$, and (2.6) and (2.8) yield that $\|T\| \leq \tau(T)$ (see [18]). Sometimes we write $\tau(T)=\tau\left(T_{A, B}\right)$ to emphasize that $T$ is considered as acting from $A$ into $B$.

A quasi-normed operator ideal is an operator ideal $\mathcal{J}$ equipped with a quasi-norm $\tau$ so that all components $\mathcal{J}(A, B)$ are complete with respect to the induced metric. If $c_{\tau}=1$, then $[\mathcal{J}, \tau]$ is said to be a normed operator ideal.

Clearly, the class of all bounded linear operators with the operator norm $[\mathcal{L},\|\cdot\|]$ is an example of a normed operator ideal. Another example is the ideal of nuclear operators $\left[\mathcal{N},\|\cdot\|_{1}\right]$. Recall that $T \in \mathcal{L}(A, B)$ is said to be nuclear if there are sequences $\left(h_{n}\right) \subset A^{*}$ and $\left(y_{n}\right) \subset B$ such that $T x=\sum_{n=1}^{\infty} h_{n}(x) y_{n}$ and $\sum_{n=1}^{\infty}\left\|h_{n}\right\|_{A^{*}}\left\|y_{n}\right\|_{B}<\infty$. The norm on $\mathcal{N}$ is given by

$$
\|T\|_{1}=\inf \left\{\sum_{n=1}^{\infty}\left\|h_{n}\right\|_{A^{*}}\left\|y_{n}\right\|_{B}\right\}
$$

where the infimum is taken over all representations $T=\sum_{n=1}^{\infty} h_{n} \otimes y_{n}$ as above. More examples will be given in Sections 4 and 5 . We refer to [18] and [4] for other details on operator ideals.

In successive sections we shall interpolate operator spaces that are components of a given ideal, so they are quasi-Banach spaces. The concept of quasi-Banach couple $\bar{X}=\left(X_{0}, X_{1}\right)$ is analogous to the Banach case, but replacing Banach spaces by quasi-Banach spaces. The $K$ - and $J$-functionals are defined in the same way. Now $X_{0} \cap X_{1}$ and $X_{0}+X_{1}$ are only quasi-Banach spaces.

If $\bar{X}=\left(X_{0}, X_{1}\right)$ is a quasi-Banach couple, $0<\theta<1$ and $0<q \leq \infty$, the real interpolation space $\bar{X}_{\theta, q}=\left(X_{0}, X_{1}\right)_{\theta, q}$ consists of all $x \in X_{0}+X_{1}$ which have a finite quasi-norm

$$
\begin{array}{ll}
\|x\|_{\theta, q}=\left(\int_{0}^{\infty}\left(t^{-\theta} K(t, x)\right)^{q} \frac{d t}{t}\right)^{1 / q} & \text { if } 0<q<\infty, \\
\|x\|_{\theta, q}=\sup _{t>0}\left\{t^{-\theta} K(t, x)\right\} & \text { if } q=\infty
\end{array}
$$

(see $[\mathbf{1}]$ or $[\mathbf{2 1}])$.

For some results we shall need to replace in the former definition the function $t^{\theta}$ by a general positive function $g:(0, \infty) \longrightarrow(0, \infty)$. The space $\bar{X}_{g, \infty ; K}=\left(X_{0}, X_{1}\right)_{g, \infty ; K}$ is formed of all $x \in X_{0}+X_{1}$ which have a finite quasi-norm

$$
\|x\|_{g, \infty ; K}=\sup _{t>0}\left\{\frac{K(t, x)}{g(t)}\right\} .
$$


It is clear that $\bar{X}_{g, \infty ; K} \hookrightarrow X_{0}+X_{1}$, but it might happen that $\bar{X}_{g, \infty ; K}=$ $\{0\}$. Indeed, for any $x \in X_{0}+X_{1}$, it follows from $\min \{1, t\}\|x\|_{X_{0}+X_{1}} \leq$ $K(t, x)$ that

$$
\sup _{t>0}\left\{\frac{\min \{1, t\}}{g(t)}\right\}\|x\|_{X_{0}+X_{1}} \leq\|x\|_{g, \infty ; K} .
$$

Consequently, the space $\bar{X}_{g, \infty ; K}$ reduces to $\{0\}$ if $\sup _{t>0}\left\{\frac{\min \{1, t\}}{g(t)}\right\}=$ $\infty$. Note that Peetre's embedding (1.1) is trivial in this case. For this reason we shall assume in the following that the function $g$ satisfies

$$
\sup _{t>0}\left\{\frac{\min \{1, t\}}{g(t)}\right\}<\infty .
$$

This condition guarantees that $X_{0} \cap X_{1} \hookrightarrow \bar{X}_{g, \infty ; K}$ because

$$
\|x\|_{g, \infty ; K}=\sup _{t>0}\left\{\frac{K(t, x)}{g(t)}\right\} \leq \sup _{t>0}\left\{\frac{\min \{1, t\}}{g(t)}\right\}\|x\|_{X_{0} \cap X_{1}} .
$$

So $\left(X_{0}, X_{1}\right)_{g, \infty ; K}$ is an intermediate space with respect to $\bar{X}$. Note that the function $\psi$ (see (2.4)) associated to $\left(X_{0}, X_{1}\right)_{g, \infty ; K}$ and $\bar{X}$ satisfies

$$
\psi(t)=\psi\left(t, \bar{X}_{g, \infty ; K} ; \bar{X}\right) \leq g(t), \quad t>0 .
$$

For $g(t)=t^{\theta}$ it holds $\bar{X}_{g, \infty ; K}=\bar{X}_{\theta, \infty}$

We shall also work with $J$-spaces. Let $f:(0, \infty) \longrightarrow(0, \infty)$ be any positive function. Assume that the constant in the triangle inequality for $X_{j}$ is $c_{j}$. Put $c=\max \left\{c_{0}, c_{1}\right\}$ and define $p$ by the equation $(2 c)^{p}=2$. If $0<q \leq p$, the space $\bar{X}_{f, q ; J}=\left(X_{0}, X_{1}\right)_{f, q ; J}$ consists of all sums $x=\sum_{m=-\infty}^{\infty} x_{m}$ (convergence in $X_{0}+X_{1}$ ) where $\left(x_{m}\right) \subset X_{0} \cap X_{1}$ and

$$
\left(\sum_{m=-\infty}^{\infty}\left(\frac{J\left(2^{m}, x_{m}\right)}{f\left(2^{m}\right)}\right)^{q}\right)^{1 / q}<\infty
$$

We put

$$
\|x\|_{f, q ; J}=\inf \left\{\left(\sum_{m=-\infty}^{\infty}\left(\frac{J\left(2^{m}, x_{m}\right)}{f\left(2^{m}\right)}\right)^{q}\right)^{\frac{1}{q}}: x=\sum_{m=-\infty}^{\infty} x_{m}\right\} .
$$

The difficulty now is that the functional $\|\cdot\|_{f, q ; J}$ is, in general, only a semi-quasi-norm. Indeed, for any $x \in X_{0} \cap X_{1}$ and any $m \in \mathbb{Z}$, we have

$$
\|x\|_{f, q ; J} \leq \frac{J\left(2^{m}, x\right)}{f\left(2^{m}\right)} \leq \frac{\max \left\{1,2^{m}\right\}}{f\left(2^{m}\right)}\|x\|_{X_{0} \cap X_{1}} .
$$


Hence

$$
\|x\|_{f, q ; J} \leq \frac{1}{\sup _{m \in \mathbb{Z}}\left\{f\left(2^{m}\right) \min \left\{1, \frac{1}{2^{m}}\right\}\right\}}\|x\|_{X_{0} \cap X_{1}} .
$$

In other words, if $\sup _{t>0}\left\{f(t) \min \left\{1, \frac{1}{t}\right\}\right\}=\infty$ then $\|x\|_{f, q ; J}=0$ for every $x \in X_{0} \cap X_{1}$. To avoid this obstruction we shall assume in the following that $f$ satisfies

$$
\sup _{t>0}\left\{f(t) \min \left\{1, \frac{1}{t}\right\}\right\}<\infty
$$

Then it is clear that $X_{0} \cap X_{1}$ is densely and continuously embedded in $\bar{X}_{f, q ; J}$. On the other hand, taking into account that the triangle inequality is valid in $X_{0}+X_{1}$ with the constant $c$, if $x=\sum_{m=-\infty}^{\infty} x_{m}$ it follows from [1, Lemma 3.10.2], that

$$
\begin{aligned}
\|x\|_{X_{0}+X_{1}} & \leq 2^{1 / p}\left(\sum_{m=-\infty}^{\infty}\left\|x_{m}\right\|_{X_{0}+X_{1}}^{p}\right)^{\frac{1}{p}} \\
& \leq 2^{1 / p}\left(\sum_{m=-\infty}^{\infty} K\left(1, x_{m}\right)^{q}\right)^{\frac{1}{q}} \\
& \leq 2^{1 / p}\left(\sum_{m=-\infty}^{\infty}\left(\min \left\{1, \frac{1}{2^{m}}\right\} J\left(2^{m}, x_{m}\right)\right)^{q}\right)^{\frac{1}{q}} \\
& \leq 2^{1 / p} \sup _{m \in \mathbb{Z}}\left\{f\left(2^{m}\right) \min \left\{1, \frac{1}{2^{m}}\right\}\right\}\left(\sum_{m=-\infty}^{\infty}\left(\frac{J\left(2^{m}, x_{m}\right)}{f\left(2^{m}\right)}\right)^{q}\right)^{\frac{1}{q}} .
\end{aligned}
$$

Since the supremum is finite by (2.11) we get that $\bar{X}_{f, q ; J} \hookrightarrow X_{0}+X_{1}$.

Let now $\rho(t)=\rho\left(t, \bar{X}_{f, q ; J} ; \bar{X}\right)$ be the function $\rho$ associated to the $J$-space $\bar{X}_{f, q ; J}$ and the couple $\bar{X}$ (see (2.5)). One can check easily that

$$
f\left(2^{m}\right) \leq \rho\left(2^{m}\right), \quad m \in \mathbb{Z} .
$$

Moreover, if $f$ is non-decreasing, it follows that

$$
f(t) \leq 2 \rho(t), \quad t>0
$$

Let us also point out that if $f(t)=t^{\theta}$ then the equivalence theorem (see [1] or [21]) yields that $\left(X_{0}, X_{1}\right)_{f, q ; J}=\left(X_{0}, X_{1}\right)_{\theta, q}$ with equivalence of quasi-norms. The reason for the restriction on $q$ in the definition of $\bar{X}_{f, q ; J}$ is that we are working with very general functions $f$. If we impose 
stronger conditions on $f$, then $\bar{X}_{f, q ; J}$ makes sense for $0<q \leq \infty$ and still is an intermediate space with respect to $\bar{X}$. The same happens with the $K$-spaces $\bar{X}_{g, q ; K}($ see $[\mathbf{1 5}])$. We observe that $\bar{X}_{g, \infty ; K}$ and $\bar{X}_{f, q ; J}$ are complete.

Given any positive function $f$, we put $f^{*}(t)=1 / f\left(t^{-1}\right)$. It turns out that $f$ satisfies (2.9) (resp. (2.11)) if and only if $f^{*}$ satisfies (2.11) (resp. (2.9)). If $f(t)=t^{\theta}$, then $f^{*}(t)=f(t)=t^{\theta}$.

Observe also that if $B$ is an intermediate space with respect to the couple $\bar{B}$, then both functions $\psi(t, B ; \bar{B})$ and $\rho(t, B ; \bar{B})$ satisfy $(2.9)$ and (2.11).

\section{Operator spaces and quasi-linearizable couples}

We start with an estimate for the norms of the operators $V_{j}(t)$ associated to a quasi-linearizable couple when they are considered as operators with $B$ as domain or $B$ as target space, where $B$ is any intermediate space.

Lemma 3.1. Let $\bar{B}=\left(B_{0}, B_{1}\right)$ be a quasi-linearizable couple, let $B$ be an intermediate space with respect to $\bar{B}$ and let $\psi(t)=\psi(t, B ; \bar{B})$ and $\rho(t)=\rho(t, B ; \bar{B})$ be the functions associated to $B$ and $\bar{B}$. Then

a) $\left\|V_{j}(t)\right\|_{B, B_{j}} \leq k t^{-j} \psi(t), j=0,1,0<t<\infty$;

b) $\left\|V_{0}(t)\right\|_{B_{1}, B} \leq(1+k) \frac{t}{\rho(t)}, 0<t<\infty$,

$\left\|V_{1}(t)\right\|_{B_{0}, B} \leq(1+k) \frac{1}{\rho(t)}, 0<t<\infty$.

Proof: Since $V_{j}(t) \in \mathcal{L}\left(B_{0}+B_{1}, B_{j}\right)$ and $B \hookrightarrow B_{0}+B_{1}$, it is clear that $V_{j}(t) \in \mathcal{L}\left(B, B_{j}\right)$. In order to estimate the norm, first note that by $(2.2)$ and (2.3)

$$
\left\|V_{j}(t) b\right\|_{B_{j}} \leq k t^{-j} K(t, b) .
$$

So, if $b \in B$ with $\|b\|_{B}=1,\left\|V_{j}(t) b\right\|_{B_{j}} \leq k t^{-j} \psi(t)$.

We go on to establish b). Assume $j=0$. By the definition of $\rho$ and properties (2.1) to (2.3), for $b \in B_{1}$ we obtain

$$
\begin{aligned}
\left\|V_{0}(t) b\right\|_{B} & \leq \frac{J\left(t, V_{0}(t) b\right)}{\rho(t)}=\frac{1}{\rho(t)} \max \left\{\left\|V_{0}(t) b\right\|_{B_{0}}, t\left\|b-V_{1}(t) b\right\|_{B_{1}}\right\} \\
& \leq(1+k) t \frac{\|b\|_{B_{1}}}{\rho(t)} .
\end{aligned}
$$

The case $j=1$ can be treated analogously.

We can now extend the result by Peetre [16, Satz 3.1]. 
Theorem 3.2. Let $\bar{B}=\left(B_{0}, B_{1}\right)$ be a quasi-linearizable couple, let $B$ be an intermediate space with respect to $\bar{B}$ and let $\psi(t)$ be the $\psi$-function associated to $B$ and $\bar{B}$. Assume $[\mathcal{J}, \tau]$ is a quasi-normed operator ideal and let $A$ be any Banach space. Then

$$
\mathcal{J}(A, B) \hookrightarrow\left(\mathcal{J}\left(A, B_{0}\right), \mathcal{J}\left(A, B_{1}\right)\right)_{\psi, \infty ; K} .
$$

Moreover, there is a constant $d$ depending only on $\bar{B}$ such that

$$
\|T\|_{\psi, \infty ; K} \leq d \tau\left(T_{A, B}\right) .
$$

Proof: Given any $T \in \mathcal{J}(A, B)$ and any $t>0$, we have by $(2.1), T=$ $V_{0}(t) T+V_{1}(t) T$ with $V_{j}(t) T \in \mathcal{J}\left(A, B_{j}\right)$ due to the ideal property of $\mathcal{J}$. Whence, using Lemma $3.1 /$ a, we derive that

$$
\begin{aligned}
\|T\|_{\psi, \infty ; K} & =\sup _{t>0}\left\{\frac{K(t, T)}{\psi(t)}\right\} \\
& \leq \sup _{t>0}\left\{\frac{\tau\left(\left[V_{0}(t) T\right]_{A, B_{0}}\right)+t \tau\left(\left[V_{1}(t) T\right]_{A, B_{1}}\right)}{\psi(t)}\right\} \\
& \leq \sup _{t>0}\left\{\frac{\tau\left(T_{A, B}\right)\left\|V_{0}(t)\right\|_{B, B_{0}}+t \tau\left(T_{A, B}\right)\left\|V_{1}(t)\right\|_{B, B_{1}}}{\psi(t)}\right\} \\
& \leq 2 k \tau\left(T_{A, B}\right) .
\end{aligned}
$$

Observe that $\left(\mathcal{J}\left(A, B_{0}\right), \mathcal{J}\left(A, B_{1}\right)\right)$ is a quasi-Banach couple because $\mathcal{J}\left(A, B_{j}\right) \hookrightarrow \mathcal{L}\left(A, B_{0}+B_{1}\right), j=0,1$. When the couple $\bar{B}$ is in the front, we need to assume that $\bar{B}$ is regular, i.e. $B_{0} \cap B_{1}$ is dense in $B_{0}$ and in $B_{1}$, in order to guarantee that $\left(\mathcal{J}\left(B_{0}, A\right), \mathcal{J}\left(B_{1}, A\right)\right)$ is a quasi-Banach couple. Indeed, under this extra supposition we have

$$
\mathcal{J}\left(B_{j}, A\right) \hookrightarrow \mathcal{L}\left(B_{j}, A\right) \hookrightarrow \mathcal{L}\left(B_{0} \cap B_{1}, A\right), \quad j=0,1 .
$$

The next result refers to this "dual" case when $\bar{B}$ is in the front.

Theorem 3.3. Let $\bar{B}=\left(B_{0}, B_{1}\right)$ be a regular quasi-linearizable couple, let $B$ be an intermediate space with respect to $\bar{B}$ with $B_{0} \cap B_{1}$ dense in $B$, and let $\rho(t)$ be the $\rho$-function associated to $B$ and $\bar{B}$. Assume $[\mathcal{J}, \tau]$ is a quasi-normed operator ideal and let $A$ be any Banach space. Then

$$
\mathcal{J}(B, A) \hookrightarrow\left(\mathcal{J}\left(B_{0}, A\right), \mathcal{J}\left(B_{1}, A\right)\right)_{\rho^{*}, \infty ; K},
$$

where $\rho^{*}(t)=1 / \rho\left(t^{-1}\right)$. Moreover, there is a constant $d$ depending only on $\bar{B}$ such that

$$
\|T\|_{\rho^{*}, \infty ; K} \leq d \tau\left(T_{B, A}\right) .
$$


Proof: Density of $B_{0} \cap B_{1}$ in $B$ implies that $\mathcal{J}(B, A)$ is also continuously embedded in $\mathcal{L}\left(B_{0} \cap B_{1}, A\right)$, so we can compare the space $\mathcal{J}(B, A)$ with $\left(\mathcal{J}\left(B_{0}, A\right), \mathcal{J}\left(B_{1}, A\right)\right)_{\rho^{*}, \infty ; K}$.

Given any $T \in \mathcal{J}(B, A)$, we can split $T$ as $T=T V_{1}(t)+T V_{0}(t)$ with $T V_{1}(t) \in \mathcal{J}\left(B_{0}, A\right)$ and $T V_{0}(t) \in \mathcal{J}\left(B_{1}, A\right)$. So, according to Lemma $3.1 / \mathrm{b}$, we get

$$
\begin{aligned}
\|T\|_{\rho^{*}, \infty ; K} & =\sup _{t>0}\left\{\rho(t) K\left(t^{-1}, T\right)\right\} \\
& \leq \sup _{t>0}\left\{\rho(t)\left[\tau\left(T_{B, A}\right)\left\|V_{1}(t)\right\|_{B_{0}, B}+t^{-1} \tau\left(T_{B, A}\right)\left\|V_{0}(t)\right\|_{B_{1}, B}\right]\right\} \\
& \leq 2(1+k) \tau\left(T_{B, A}\right) .
\end{aligned}
$$

Assume now that $g:(0, \infty) \longrightarrow(0, \infty)$ is a function satisfying $(2.9)$ and choose $B=\left(B_{0}, B_{1}\right)_{g, \infty ; K}$. As we pointed out in $(2.10), \psi(t, B ; \bar{B}) \leq$ $g(t)$ for $t>0$. Hence

$$
\left(\mathcal{J}\left(A, B_{0}\right), \mathcal{J}\left(A, B_{1}\right)\right)_{\psi, \infty ; K} \hookrightarrow\left(\mathcal{J}\left(A, B_{0}\right), \mathcal{J}\left(A, B_{1}\right)\right)_{g, \infty ; K}
$$

This remark allows us to derive from Theorem 3.2 the following consequence:

Corollary 3.4. Let $\bar{B}=\left(B_{0}, B_{1}\right)$ be a quasi-linearizable couple and let $g$ be a positive function satisfying (2.9). If $[\mathcal{J}, \tau]$ is any quasi-normed operator ideal and $A$ is any Banach space, then

$$
\mathcal{J}\left(A,\left(B_{0}, B_{1}\right)_{g, \infty ; K}\right) \hookrightarrow\left(\mathcal{J}\left(A, B_{0}\right), \mathcal{J}\left(A, B_{1}\right)\right)_{g, \infty ; K} .
$$

Moreover, there is a constant $d$ depending only on $\bar{B}$ such that

$$
\|T\|_{g, \infty ; K} \leq d \tau\left(T_{A, \bar{B}_{g, \infty ; K}}\right) .
$$

On the other hand, Theorem 3.3 and (2.13) yield:

Corollary 3.5. Let $\bar{B}=\left(B_{0}, B_{1}\right)$ be a regular quasi-linearizable couple and let $f$ be a non-decreasing positive function satisfying (2.11). If $[\mathcal{J}, \tau]$ is any quasi-normed operator ideal and $A$ is any Banach space, then

$$
\mathcal{J}\left(\left(B_{0}, B_{1}\right)_{f, 1 ; J}, A\right) \hookrightarrow\left(\mathcal{J}\left(B_{0}, A\right), \mathcal{J}\left(B_{1}, A\right)\right)_{f^{*}, \infty ; K}
$$

Moreover, there exists a constant d depending only on $\bar{B}$ such that

$$
\|T\|_{f^{*}, \infty ; K} \leq d \tau\left(T_{\bar{B}_{f, 1 ; J}, A}\right)
$$

Writing down Corollary 3.4 for the ideal $\mathcal{J}=\mathcal{L}$ of all bounded linear operators we recover Peetre's result [16, Satz 3.1] (recall that, as we pointed out in Section 2, the embedding (1.1) is trivial if condition (2.9) is 
not satisfied). Peetre proved in [16, Satz 3.2], that the validity of Corollary 3.4 for $\mathcal{J}=\mathcal{L}$ forces $\bar{B}$ to be quasi-linearizable. Hence without this assumption Theorem 3.2 and Corollary 3.4 are not true in general. Next we show that the same happens with Theorem 3.3 and Corollary 3.5.

Theorem 3.6. Let $\bar{B}=\left(B_{0}, B_{1}\right)$ be a regular Banach couple. If there is a constant $d$ such that for any Banach space $A$ and any non-decreasing positive function $f$ satisfying (2.11) it holds

$$
\mathcal{L}\left(\left(B_{0}, B_{1}\right)_{f, 1 ; J}, A\right) \hookrightarrow\left(\mathcal{L}\left(B_{0}, A\right), \mathcal{L}\left(B_{1}, A\right)\right)_{f^{*}, \infty ; K}
$$

with

$$
\|T\|_{f^{*}, \infty ; K} \leq d\|T\|_{\bar{B}_{f, 1 ; J}, A},
$$

then $\bar{B}$ must be quasi-linearizable.

Proof: Fix $s>0$ and put $f(t)=\min \left\{1, \frac{t}{s}\right\}$. Then $f$ is non-decreasing, satisfies (2.11) and $\bar{B}_{f, 1 ; J}=\left(B_{0} \cap B_{1}, J(s, \cdot)\right)$ with

$$
J(s, b) \leq\|b\|_{f, 1 ; J} \leq 2 J(s, b) .
$$

Indeed, if $b=\sum_{m=-\infty}^{\infty} b_{m}$ is any $J$-representation of $b$, we have

$$
\begin{aligned}
J(s, b) & \leq \sum_{m=-\infty}^{\infty} J\left(s, b_{m}\right) \\
& \leq \sum_{m=-\infty}^{\infty} \max \left\{1, \frac{s}{2^{m}}\right\} J\left(2^{m}, b_{m}\right)=\sum_{m=-\infty}^{\infty} \frac{J\left(2^{m}, b_{m}\right)}{f\left(2^{m}\right)} .
\end{aligned}
$$

This gives the first inequality in (3.1). The second one follows from the choice

$$
b_{m}= \begin{cases}0, & \text { if } m \neq n \\ b, & \text { if } m=n\end{cases}
$$

where $2^{n} \leq s<2^{n+1}$, because

$$
\|b\|_{f, 1 ; J} \leq \frac{J\left(2^{n}, b\right)}{f\left(2^{n}\right)} \leq \frac{s}{2^{n}} J(s, b) \leq 2 J(s, b) .
$$

Take next $A=\bar{B}_{f, 1 ; J}$ and $T=I_{\bar{B}_{f, 1 ; J}}$ the identity mapping of $\bar{B}_{f, 1 ; J}$. According to our assumption, there are operators $V_{1}(s) \in \mathcal{L}\left(B_{0}, \bar{B}_{f, 1 ; J}\right)$ and $V_{0}(s) \in \mathcal{L}\left(B_{1}, \bar{B}_{f, 1 ; J}\right)$ such that

$$
V_{0}(s)+V_{1}(s)=T=I_{B_{0} \cap B_{1}}
$$


and

$$
\begin{aligned}
\left\|V_{1}(s)\right\|_{B_{0}, \bar{B}_{f, 1 ; J}}+s^{-1}\left\|V_{0}(s)\right\|_{B_{1}, \bar{B}_{f, 1 ; J}} & <2 K\left(s^{-1}, T\right) \leq 2 \sup _{t>0} \frac{K(t, T)}{f^{*}(t)} \\
& \leq 2 d\|T\|_{\bar{B}_{f, 1 ; J}, \bar{B}_{f, 1 ; J}}=2 d .
\end{aligned}
$$

Therefore

$$
\begin{array}{ll}
\left\|V_{0}(s) b\right\|_{B_{0}} \leq J\left(s, V_{0}(s) b\right) \leq 2 d s\|b\|_{B_{1}}, & b \in B_{1}, \\
\left\|V_{0}(s) b\right\|_{B_{1}} \leq s^{-1} J\left(s, V_{0}(s) b\right) \leq 2 d\|b\|_{B_{1}}, & b \in B_{1},
\end{array}
$$

and similarly

$$
\begin{aligned}
\left\|V_{1}(s) b\right\|_{B_{1}} \leq 2 d s^{-1}\|b\|_{B_{0}}, & b \in B_{0}, \\
\left\|V_{1}(s) b\right\|_{B_{0}} \leq 2 d\|b\|_{B_{0}}, & b \in B_{0} .
\end{aligned}
$$

Thus, if $b \in B_{0} \cap B_{1}$, we obtain

$$
\left\|V_{0}(s) b\right\|_{B_{0}} \leq\|b\|_{B_{0}}+\left\|V_{1}(s) b\right\|_{B_{0}} \leq(1+2 d)\|b\|_{B_{0}}
$$

and in the same way

$$
\left\|V_{1}(s) b\right\|_{B_{1}} \leq(1+2 d)\|b\|_{B_{1}} .
$$

Taking into account that $\bar{B}$ is regular, and so $B_{0} \cap B_{1}$ is dense in $B_{0}+B_{1}$, as well, it is not hard to check that the operators $V_{j}(s)$ can be extended boundedly to operators (denoted by the same letters) $V_{j}(s) \in$ $\mathcal{L}\left(B_{0}+B_{1}, B_{j}\right)$ such that $(3.2)$ holds for any $b \in B_{0}$, (3.3) for any $b \in B_{1}$ and $V_{0}(s)+V_{1}(s)=I$ in $B_{0}+B_{1}$. The proof is complete.

The remainder of this section is devoted to interpolation by the $J$-method. We denote by $c_{\tau}$ the constant in the quasi-triangle inequality (2.7).

Theorem 3.7. Let $\bar{B}=\left(B_{0}, B_{1}\right)$ be a quasi-linearizable couple, let $B$ be an intermediate space with respect to $\bar{B}$ and let $\rho(t)$ be the $\rho$-function associated to $B$ and $\bar{B}$. Assume that $A$ is any Banach space, that $[\mathcal{J}, \tau]$ is a quasi-normed operator ideal and let $q$ be defined by the equation $\left(2 c_{\tau}\right)^{q}=2$. Then

$$
\left(\mathcal{J}\left(A, B_{0}\right), \mathcal{J}\left(A, B_{1}\right)\right)_{\rho, q ; J} \hookrightarrow \mathcal{J}(A, B) .
$$

Proof: Let $T \in \mathcal{J}\left(A, B_{0}\right) \cap \mathcal{J}\left(A, B_{1}\right)$. The decomposition

$$
T=V_{1}(t) T+V_{0}(t) T
$$


yields that $T \in \mathcal{J}(A, B)$ with

$$
\begin{aligned}
\tau\left(T_{A, B}\right) & \leq c_{\tau}\left(\left\|V_{1}(t)\right\|_{B_{0}, B} \tau\left(T_{A, B_{0}}\right)+\left\|V_{0}(t)\right\|_{B_{1}, B} \tau\left(T_{A, B_{1}}\right)\right) \\
& \leq c_{\tau}(1+k) \frac{1}{\rho(t)}\left(\tau\left(T_{A, B_{0}}\right)+t \tau\left(T_{A, B_{1}}\right)\right) \\
& \leq 2 c_{\tau}(1+k) \frac{1}{\rho(t)} J(t, T),
\end{aligned}
$$

where we have used again Lemma 3.1 to estimate $\left\|V_{1}(t)\right\|_{B_{0}, B}$ and $\left\|V_{0}(t)\right\|_{B_{1}, B}$. Suppose now that $T \in\left(\mathcal{J}\left(A, B_{0}\right), \mathcal{J}\left(A, B_{1}\right)\right)_{\rho, q ; J}$ and let $T=\sum_{m=-\infty}^{\infty} T_{m}$ be any $J$-representation of $T$. Using [1, Lemma 3.10.2] and the previous estimates, we obtain that

$$
\begin{aligned}
\tau\left(T_{A, B}\right) & \leq 2^{\frac{1}{q}}\left(\sum_{m=-\infty}^{\infty}\left(\tau\left(T_{m}\right)\right)^{q}\right)^{\frac{1}{q}} \\
& \leq 2^{\frac{1}{q}} 2 c_{\tau}(1+k)\left(\sum_{m=-\infty}^{\infty}\left(\frac{J\left(2^{m}, T_{m}\right)}{\rho\left(2^{m}\right)}\right)^{q}\right)^{\frac{1}{q}} .
\end{aligned}
$$

Consequently, $T \in \mathcal{J}(A, B)$ with $\tau\left(T_{A, B}\right) \leq 2^{\frac{1}{q}} 2 c_{\tau}(1+k)\|T\|_{\rho, q ; J}$.

Theorem 3.8. Let $\bar{B}=\left(B_{0}, B_{1}\right)$ be a regular quasi-linearizable couple, let $B$ be an intermediate space with respect to $\bar{B}$ with $B_{0} \cap B_{1}$ dense in $B$ and let $\psi(t)$ be the $\psi$-function associated to $B$ and $\bar{B}$. If $A$ is any Banach space, $[\mathcal{J}, \tau]$ is a quasi-normed operator ideal and $q$ is defined by $\left(2 c_{\tau}\right)^{q}=2$, then

$$
\left(\mathcal{J}\left(B_{0}, A\right), \mathcal{J}\left(B_{1}, A\right)\right)_{\psi^{*}, q ; J} \hookrightarrow \mathcal{J}(B, A) .
$$

Proof: Let $T \in \mathcal{J}\left(B_{0}, A\right) \cap \mathcal{J}\left(B_{1}, A\right)$ and decompose it in the way $T=$ $T V_{0}(t)+T V_{1}(t)$. It is clear that $T \in \mathcal{J}(B, A)$ with

$$
\begin{aligned}
\tau\left(T_{B, A}\right) & \leq c_{\tau}\left[\tau\left(T_{B_{0}, A}\right)\left\|V_{0}(t)\right\|_{B, B_{0}}+\tau\left(T_{B_{1}, A}\right)\left\|V_{1}(t)\right\|_{B, B_{1}}\right] \\
& \leq c_{\tau} k \psi(t)\left[\tau\left(T_{B_{0}, A}\right)+\frac{1}{t} \tau\left(T_{B_{1}, A}\right)\right] \leq 2 c_{\tau} k \frac{J\left(t^{-1}, T\right)}{\psi^{*}\left(t^{-1}\right)} .
\end{aligned}
$$

Now the result follows by combining this estimate with [1, Lemma 3.10.2] as we did in Theorem 3.7.

The next result follows from Theorems 3.7 and 3.8 by using (2.10) and (2.12). We put $\left(B_{0}, B_{1}\right)_{g, \infty ; K}^{o}$ for the clousure of $B_{0} \cap B_{1}$ in $\left(B_{0}, B_{1}\right)_{g, \infty ; K}$. 
Corollary 3.9. Let $\bar{B}=\left(B_{0}, B_{1}\right)$ be a quasi-linearizable couple and let $A$ be a Banach space. Suppose that $[\mathcal{J}, \tau]$ is a quasi-normed operator ideal and defined $q$ by $\left(2 c_{\tau}\right)^{q}=2$.

a) If $f$ satisfies (2.11), then

$$
\left(\mathcal{J}\left(A, B_{0}\right), \mathcal{J}\left(A, B_{1}\right)\right)_{f, q ; J} \hookrightarrow \mathcal{J}\left(A,\left(B_{0}, B_{1}\right)_{f, 1 ; J}\right) .
$$

b) If $\bar{B}$ is regular and $g$ satisfies (2.9), then

$$
\left(\mathcal{J}\left(B_{0}, A\right), \mathcal{J}\left(B_{1}, A\right)\right)_{g^{*}, q ; J} \hookrightarrow \mathcal{J}\left(\left(B_{0}, B_{1}\right)_{g, \infty ; K}^{o}, A\right) .
$$

\section{Some applications}

We begin identifying some interpolation spaces.

Theorem 4.1. Let $\bar{B}=\left(B_{0}, B_{1}\right)$ be a quasi-linearizable couple and let $A$ be a Banach space.

a) If $g$ is positive and satisfies (2.9), then

$$
\mathcal{L}\left(A,\left(B_{0}, B_{1}\right)_{g, \infty ; K}\right)=\left(\mathcal{L}\left(A, B_{0}\right), \mathcal{L}\left(A, B_{1}\right)\right)_{g, \infty ; K}
$$

(equivalent norms).

b) If $\bar{B}$ is regular and $f$ is a non-decreasing positive function satisfying (2.11) then

$$
\mathcal{L}\left(\left(B_{0}, B_{1}\right)_{f, 1 ; J}, A\right)=\left(\mathcal{L}\left(B_{0}, A\right), \mathcal{L}\left(B_{1}, A\right)\right)_{f^{*}, \infty ; K}
$$

(equivalent norms).

Proof: First we prove b). By Corollary 3.5, we have that $\mathcal{L}\left(\bar{B}_{f, 1 ; J}, A\right) \hookrightarrow$ $\left(\mathcal{L}\left(B_{0}, A\right), \mathcal{L}\left(B_{1}, A\right)\right)_{f^{*}, \infty ; K}$. In order to establish the converse inclusion let $T \in\left(\mathcal{L}\left(B_{0}, A\right), \mathcal{L}\left(B_{1}, A\right)\right)_{f^{*}, \infty ; K}$ and $m \in \mathbb{Z}$. Since $K\left(2^{m}, T\right) \leq$ $f^{*}\left(2^{m}\right)\|T\|_{f^{*}, \infty ; K}$ we can find a decomposition $T=T_{0}^{m}+T_{1}^{m}$ of $T$ with $T_{j}^{m} \in \mathcal{L}\left(B_{j}, A\right)$ and

$$
\left\|T_{0}^{m}\right\|_{B_{0}, A}+2^{m}\left\|T_{1}^{m}\right\|_{B_{1}, A} \leq 2 f^{*}\left(2^{m}\right)\|T\|_{f^{*}, \infty ; K} .
$$

Then, for any $b \in B_{0} \cap B_{1}$, it follows that

$$
\begin{aligned}
\|T b\|_{A} & \leq\left\|T_{0}^{m} b\right\|_{A}+\left\|T_{1}^{m} b\right\|_{A} \leq\left\|T_{0}^{m}\right\|_{B_{0}, A}\|b\|_{B_{0}}+2^{m}\left\|T_{1}^{m}\right\|_{B_{1}, A} \frac{\|b\|_{B_{1}}}{2^{m}} \\
& \leq\left(\left\|T_{0}^{m}\right\|_{B_{0}, A}+2^{m}\left\|T_{1}^{m}\right\|_{B_{1}, A}\right) J\left(2^{-m}, b\right) \\
& \leq 2 f^{*}\left(2^{m}\right)\|T\|_{f^{*}, \infty ; K} J\left(2^{-m}, b\right)=2\|T\|_{f^{*}, \infty ; K} \frac{J\left(2^{-m}, b\right)}{f\left(2^{-m}\right)}
\end{aligned}
$$


If now $b \in \bar{B}_{f, 1 ; J}$ and $b=\sum_{m=-\infty}^{\infty} b_{m}$ is a $J$-representation of $b$, we get

$$
\|T b\|_{A} \leq \sum_{m=-\infty}^{\infty}\left\|T b_{m}\right\|_{A} \leq 2\|T\|_{f^{*}, \infty ; K} \sum_{m=-\infty}^{\infty} \frac{J\left(2^{m}, b_{m}\right)}{f\left(2^{m}\right)}
$$

This implies $\|T\|_{\bar{B}_{f, 1 ; J}, A} \leq 2\|T\|_{f^{*}, \infty ; K}$ and ends the proof of b). The proof of a) is more easy. The inclusion $\left(\mathcal{L}\left(A, B_{0}\right), \mathcal{L}\left(A, B_{1}\right)\right)_{g, \infty ; K} \hookrightarrow$ $\mathcal{L}\left(A,\left(B_{0}, B_{1}\right)_{g, \infty ; K}\right)$ follows from a similar argument to the one given before, but that now can be simplified because we work with $K$-spaces only. The converse inclusion follows from Corollary 3.4.

Our next result deals with nuclear operators.

Theorem 4.2. Let $\bar{B}=\left(B_{0}, B_{1}\right)$ be a quasi-linearizable couple, let $A$ be a Banach space and assume that $f$ is a positive function satisfying (2.11). Then

$$
\mathcal{N}\left(A,\left(B_{0}, B_{1}\right)_{f, 1 ; J}\right)=\left(\mathcal{N}\left(A, B_{0}\right), \mathcal{N}\left(A, B_{1}\right)\right)_{f, 1 ; J}
$$

(equivalent norms).

Proof: As we pointed out in Section $2,\left[\mathcal{N},\|\cdot\|_{1}\right]$ is a normed operator ideal so we can apply Corollary $3.9 /$ a with $q=1$. We obtain that

$$
\left(\mathcal{N}\left(A, B_{0}\right), \mathcal{N}\left(A, B_{1}\right)\right)_{f, 1 ; J} \hookrightarrow \mathcal{N}\left(A,\left(B_{0}, B_{1}\right)_{f, 1 ; J}\right) .
$$

Conversely, let $T \in \mathcal{N}\left(A, \bar{B}_{f, 1 ; J}\right)$. We can find sequences $\left(h_{n}\right) \subset A^{*}$ and $\left(b_{n}\right) \subset \bar{B}_{f, 1 ; J}$ such that

$$
T=\sum_{n=1}^{\infty} h_{n} \otimes b_{n} \quad \text { and } \quad \sum_{n=1}^{\infty}\left\|h_{n}\right\|_{A^{*}}\left\|b_{n}\right\|_{f, 1 ; J} \leq 2\|T\|_{1} .
$$

On the other hand, each $b_{n}$ can be written as $b_{n}=\sum_{m=-\infty}^{\infty} b_{m}^{n}$ with $\left(b_{m}^{n}\right) \subset B_{0} \cap B_{1}$ and

$$
\sum_{m=-\infty}^{\infty} \frac{J\left(2^{m}, b_{m}^{n}\right)}{f\left(2^{m}\right)} \leq 2\left\|b_{n}\right\|_{f, 1 ; J}
$$

Put $S_{n}=\sum_{m=-\infty}^{\infty} h_{n} \otimes b_{m}^{n}$. For each $n \in \mathbb{N}$, the operator $S_{n}$ belongs to the space $\left(\mathcal{N}\left(A, B_{0}\right), \mathcal{N}\left(A, B_{1}\right)_{f, 1 ; J}\right.$, because

$$
\sum_{m=-\infty}^{\infty} \frac{J\left(2^{m}, h_{n} \otimes b_{m}^{n}\right)}{f\left(2^{m}\right)} \leq 2\left\|h_{n}\right\|_{A^{*}}\left\|b_{n}\right\|_{f, 1 ; J}
$$


Since $T=\sum_{n=1}^{\infty} S_{n}$, we conclude that $T \in\left(\mathcal{N}\left(A, B_{0}\right), \mathcal{N}\left(A, B_{1}\right)\right)_{f, 1 ; J}$ with

$$
\|T\|_{f, 1 ; J} \leq \sum_{n=1}^{\infty}\left\|S_{n}\right\|_{f, 1 ; J} \leq 2 \sum_{n=1}^{\infty}\left\|h_{n}\right\|_{A^{*}}\left\|b_{n}\right\|_{f, 1 ; J} \leq 4\|T\|_{1} .
$$

The proof is complete.

Assume next that the function $g$ belongs to the class $\mathcal{P}^{+-}$(see [10]). This means that $g(t)$ is non-decreasing, $g(t) / t$ is non-increasing with $\bar{g}(t)=\sup _{s>0}\{g(t s) / g(s)\}$ finite for every $t>0$ and $\bar{g}(t)=o(\max \{1, t\})$ as $t \rightarrow 0$ and $t \rightarrow \infty$. Then it is well-known that the spaces $\left(B_{0}, B_{1}\right)_{g, q ; K}$ and $\left(B_{0}, B_{1}\right)_{g, q ; J}$ coincide, and that for regular couples the following duality formula holds

$$
\left(\left(B_{0}, B_{1}\right)_{g, \infty ; K}^{o}\right)^{*}=\left(B_{0}^{*}, B_{1}^{*}\right)_{g^{*}, 1 ; J}
$$

Arguing as in Theorem 4.2 but using Corollary 3.9/b, we obtain

Theorem 4.3. Let $\bar{B}=\left(B_{0}, B_{1}\right)$ be a regular quasi-linearizable couple, let $A$ be a Banach space and assume that $g \in \mathcal{P}^{+-}$. Then

$$
\mathcal{N}\left(\left(B_{0}, B_{1}\right)_{g, \infty ; K}^{o}, A\right)=\left(\mathcal{N}\left(B_{0}, A\right), \mathcal{N}\left(B_{1}, A\right)\right)_{g^{*}, 1 ; J}
$$

(equivalent norms).

Previous results in this direction are due to Ovchinnikov [13] and [14]. His approach works for regular couples of Hilbert spaces $\left(H_{0}, H_{1}\right)$, $\left(G_{0}, G_{1}\right)$ and gives that

$$
\begin{aligned}
\left(\mathcal{L}\left(H_{0}, G_{0}\right), \mathcal{L}\left(H_{1}, G_{1}\right)\right)_{\theta, \infty} & =\mathcal{L}\left(\left(H_{0}, H_{1}\right)_{\theta, 1},\left(G_{0}, G_{1}\right)_{\theta, \infty}\right), \\
\left(\mathcal{N}\left(H_{0}, G_{0}\right), \mathcal{N}\left(H_{1}, G_{1}\right)\right)_{\theta, 1} & =\mathcal{N}\left(\left(H_{0}, H_{1}\right)_{\theta, \infty}^{o},\left(G_{0}, G_{1}\right)_{\theta, 1}\right) .
\end{aligned}
$$

Note that each one of the couples $\left(H_{0}, H_{1}\right),\left(G_{0}, G_{1}\right)$ is quasi-linearizable. The reason is that any regular couple of Hilbert spaces is isomorphic to a couple $\left(\ell_{2}\left(G_{m}\right), \ell_{2}\left(2^{-m} G_{m}\right)\right)$ formed by vector valued $\ell_{2}$-spaces (see $[\mathbf{6}]$ ), so the quasi-linearizability follows from the next result.

Lemma 4.4. Let $1 \leq p \leq \infty$ and let $\left(G_{m}\right)_{m \in \mathbb{Z}}$ be a sequence of Banach spaces. Then the couple $\left(\ell_{p}\left(G_{m}\right), \ell_{p}\left(2^{-m} G_{m}\right)\right)$ is quasi-linearizable. 
Proof: Let $x=\left(x_{m}\right)_{m \in \mathbb{Z}} \in \ell_{p}\left(G_{m}\right)+\ell_{p}\left(2^{-m} G_{m}\right)$. Then

$$
\begin{gathered}
K(t, x)^{p} \approx \inf \left\{\sum_{m=-\infty}^{\infty}\left\|y_{m}\right\|_{G_{m}}^{p}+t^{p} \sum_{m=-\infty}^{\infty} 2^{-m p}\left\|z_{m}\right\|_{G_{m}}^{p}:\right. \\
\left.x=y+z, y \in \ell_{p}\left(G_{m}\right), z \in \ell_{p}\left(2^{-m} G_{m}\right)\right\} \\
=\sum_{m=-\infty}^{\infty} \min \left\{1, t^{p} 2^{-m p}\right\}\left\|x_{m}\right\|_{G_{m}}^{p} \\
=\sum_{m \in Z_{1}}\left\|x_{m}\right\|_{G_{m}}^{p}+t^{p} \sum_{m \in Z_{2}} 2^{-m p}\left\|x_{m}\right\|_{G_{m}}^{p}
\end{gathered}
$$

where $Z_{1}=\left\{m \in \mathbb{Z}: 2^{m} \leq t\right\}$ and $Z_{2}=\mathbb{Z} \backslash Z_{1}$. This suggest to define for each $t>0$

$$
V_{0}(t) x=\left(v_{m}\right), \quad V_{1}(t) x=\left(w_{m}\right)
$$

where

$$
v_{m}=\left\{\begin{array}{ll}
x_{m} & \text { if } m \in Z_{1} \\
0 & \text { otherwise }
\end{array}, \quad w_{m}=\left\{\begin{array}{ll}
0 & \text { if } m \in Z_{1} \\
x_{m} & \text { otherwise }
\end{array} .\right.\right.
$$

A direct computation shows that conditions (2.1), (2.2) and (2.3) are fulfilled with $k=1$.

It is well-known the relationship between $\mathcal{N}(A, B)$ and the projective tensor product $A^{*} \hat{\otimes} B$, so one may guess that the results of Section 3 are also useful to interpolate projective tensor products, or even injective tensor products $A \check{\otimes} B$. In fact, by means of similar arguments to those described in Theorems 3.2 and 4.1, one can establish

Theorem 4.5. Let $\bar{B}=\left(B_{0}, B_{1}\right)$ be a regular quasi-linearizable couple, let $A$ be a Banach space and assume that $g \in \mathcal{P}^{+-}$. Then

$$
A \check{\otimes}\left(B_{0}, B_{1}\right)_{g, \infty ; K}^{o}=\left(A \check{\otimes} B_{0}, A \check{\otimes} B_{1}\right)_{g, \infty ; K}^{o}
$$

(equivalent norms).

The result for the projective tensor product reads

Theorem 4.6. Let $\bar{B}=\left(B_{0}, B_{1}\right)$ be a regular quasi-linearizable couple and let $A$ be a Banach space such that $\left(A \hat{\otimes} B_{0}, A \hat{\otimes} B_{1}\right)$ is also a Banach couple. If $f$ is any positive function satisfying (2.11), then

$$
A \hat{\otimes}\left(B_{0}, B_{1}\right)_{f, 1 ; J}=\left(A \hat{\otimes} B_{0}, A \hat{\otimes} B_{1}\right)_{f, 1 ; J}
$$

(equivalent norms). 
The proof follows similar lines to those in Theorem 4.2.

Previous results on interpolation of tensor products are due to Kouba [11]. He found conditions on the regular Banach couples $\left(A_{0}, A_{1}\right)$ and $\left(B_{0}, B_{1}\right)$ so that the following complex interpolation formulæ

$$
\begin{aligned}
& {\left[A_{0}, A_{1}\right]_{\theta} \check{\otimes}\left[B_{0}, B_{1}\right]_{\theta}=\left[A_{0} \check{\otimes} B_{0}, A_{1} \check{\otimes} B_{1}\right]_{\theta},} \\
& {\left[A_{0}, A_{1}\right]_{\theta} \hat{\otimes}\left[B_{0}, B_{1}\right]_{\theta}=\left[A_{0} \hat{\otimes} B_{0}, A_{1} \hat{\otimes} B_{1}\right]_{\theta}}
\end{aligned}
$$

are valid. His conditions has nothing to be with the quasi-linearizability, but they refers to the notions of type 2 and 2-convexity.

Next we focus our attention on operator ideals defined by approximation numbers. Recall that for $T \in \mathcal{L}(A, B)$ and $n=1,2, \ldots$, the $n$-th approximation number is given by

$$
a_{n}(T)=a_{n}\left(T_{A, B}\right)=\inf \{\|T-L\|: L \in \mathcal{L}(A, B), \operatorname{rank} L<n\} .
$$

For $0<p<\infty$, the quasi-normed operator ideals $\left[\mathcal{L}_{p}^{(a)}, \sigma_{p}\right]$ generated by the approximation numbers and the sequence space $\ell_{p}$ are defined by

$$
\mathcal{L}_{p}^{(a)}(A, B)=\left\{T \in \mathcal{L}(A, B): \sigma_{p}(T)=\left(\sum_{n=1}^{\infty} a_{n}(T)^{p}\right)^{\frac{1}{p}}<\infty\right\}
$$

(see $[\mathbf{1 8}]$ and $[\mathbf{1 9}]$ ).

It follows from [8, Theorem 3.3.4], that approximation numbers have no unrestricted interpolation properties. However, working with quasilinearizable couples, we can use ideas of Section 3 to establish the following formulæ:

Lemma 4.7. Let $A$ be a Banach space, let $\bar{B}=\left(B_{0}, B_{1}\right)$ be a quasilinearizable couple, let $B$ be an intermediate space with respect to $\bar{B}$ and let $\psi(t)$ and $\rho(t)$ be the functions associated to $B$ and $\bar{B}$.

a) If $T \in \mathcal{L}\left(A, B_{0} \cap B_{1}\right)$ and $n_{0}, n_{1} \in \mathbb{N}$, then

$$
a_{n_{0}+n_{1}-1}\left(T_{A, B}\right) \leq 2(1+k) a_{n_{0}}\left(T_{A, B_{0}}\right) \rho^{*}\left(\frac{a_{n_{1}}\left(T_{A, B_{1}}\right)}{a_{n_{0}}\left(T_{A, B_{0}}\right)}\right) .
$$

b) If $\bar{B}$ is regular, $T \in \mathcal{L}\left(B_{0}+B_{1}, A\right)$ and $n_{0}, n_{1} \in \mathbb{N}$, then

$$
a_{n_{0}+n_{1}-1}\left(T_{B, A}\right) \leq 2 k a_{n_{0}}\left(T_{B_{0}, A}\right) \psi\left(\frac{a_{n_{1}}\left(T_{B_{1}, A}\right)}{a_{n_{0}}\left(T_{B_{0}, A}\right)}\right) .
$$


Proof: Let $T \in \mathcal{L}\left(A, B_{0} \cap B_{1}\right)$. Splitting $T$ as $T=V_{1}(t) T+V_{0}(t) T$, using Lemma 3.1 and additivity of approximation numbers, we get that

$$
\begin{aligned}
a_{n_{0}+n_{1}-1}\left(T_{A, B}\right) & \leq a_{n_{0}}\left(\left[V_{1}(t) T\right]_{A, B}\right)+a_{n_{1}}\left(\left[V_{0}(t) T\right]_{A, B}\right) \\
& \leq\left\|V_{1}(t)\right\|_{B_{0}, B} a_{n_{0}}\left(T_{A, B_{0}}\right)+\left\|V_{0}(t)\right\|_{B_{1}, B} a_{n_{1}}\left(T_{A, B_{1}}\right) \\
& \leq(1+k)\left[\frac{a_{n_{0}}\left(T_{A, B_{0}}\right)}{\rho(t)}+\frac{t a_{n_{1}}\left(T_{A, B_{1}}\right)}{\rho(t)}\right] .
\end{aligned}
$$

If $a_{n_{j}}\left(T_{A, B_{j}}\right) \neq 0$, for $j=0,1$, then the choice $t=a_{n_{0}}\left(T_{A, B_{0}}\right) / a_{n_{1}}\left(T_{A, B_{1}}\right)$ yields a). If $a_{n_{j}}\left(T_{A, B_{j}}\right)=0$, for $j=0$ or $j=1$, then $a_{n_{0}+n_{1}-1}\left(T_{A, B}\right)=0$ as well, because rank $T<n_{j}$. The proof of b) is similar, using now the splitting $T=T V_{0}(t)+T V_{1}(t)$.

See [7] for some remarks on the connection between this result and the study of approximation numbers of embeddings from Besov spaces into spaces of Lipschitz type.

We are now ready to establish embedding formulæ for $\mathcal{L}_{p}^{(a)}$-ideals.

Theorem 4.8. Let $A$ be a Banach space and let $\bar{B}=\left(B_{0}, B_{1}\right)$ be a quasi-linearizable couple. Assume that $0<p_{0}, p_{1}<\infty, 0<\theta<1$, $\frac{1}{p}=\frac{1-\theta}{p_{0}}+\frac{\theta}{p_{1}}$ and $\frac{1}{q}=\max \left\{1, \frac{1}{p}\right\}+\frac{1}{p}$. Then the following holds:

a) $\left(\mathcal{L}_{p_{0}}^{(a)}\left(A, B_{0}\right), \mathcal{L}_{p_{1}}^{(a)}\left(A, B_{1}\right)\right)_{\theta, q} \hookrightarrow \mathcal{L}_{p}^{(a)}\left(A,\left(B_{0}, B_{1}\right)_{\theta, 1}\right)$.

b) Moreover, if $\bar{B}$ is regular,

$$
\left(\mathcal{L}_{p_{0}}^{(a)}\left(B_{0}, A\right), \mathcal{L}_{p_{1}}^{(a)}\left(B_{1}, A\right)\right)_{\theta, q} \hookrightarrow \mathcal{L}_{p}^{(a)}\left(\left(B_{0}, B_{1}\right)_{\theta, \infty}^{o}, A\right) .
$$

Proof: Let $T \in \mathcal{L}_{p_{0}}^{(a)}\left(A, B_{0}\right) \cap \mathcal{L}_{p_{1}}^{(a)}\left(A, B_{1}\right)$. Using Lemma $4.7 /$ a with $B=\left(B_{0}, B_{1}\right)_{\theta, 1}=\left(B_{0}, B_{1}\right)_{f, 1 ; J}$ where $f(t)=t^{\theta}$, we have that

$$
a_{2 n-1}\left(T_{A,\left(B_{0}, B_{1}\right)_{\theta, 1}}\right) \leq 2^{1+\theta}(1+k) a_{n}\left(T_{A, B_{0}}\right)^{1-\theta} a_{n}\left(T_{A, B_{1}}\right)^{\theta} .
$$

Whence

$$
\begin{aligned}
\sigma_{p}\left(T_{A,\left(B_{0}, B_{1}\right)_{\theta, 1}}\right) & \leq 2^{\frac{1}{p}}\left(\sum_{n=1}^{\infty} a_{2 n-1}\left(T_{A,\left(B_{0}, B_{1}\right)_{\theta, 1}}\right)^{p}\right)^{\frac{1}{p}} \\
& \leq 2^{\frac{\theta p+p+1}{p}}(1+k)\left(\sum_{n=1}^{\infty} a_{n}\left(T_{A, B_{0}}\right)^{(1-\theta) p} a_{n}\left(T_{A, B_{1}}\right)^{\theta p}\right)^{\frac{1}{p}} \\
& \leq 2^{\frac{\theta p+p+1}{p}}(1+k) \sigma_{p_{0}}\left(T_{A, B_{0}}\right)^{1-\theta} \sigma_{p_{1}}\left(T_{A, B_{1}}\right)^{\theta} \\
& \leq 2^{\frac{\theta p+p+1}{p}}(1+k) 2^{-\theta m} J\left(2^{m}, T\right)
\end{aligned}
$$

where $m \in \mathbb{Z}$ is arbitrary. 
Take now $T \in\left(\mathcal{L}_{p_{0}}^{(a)}\left(A, B_{0}\right), \mathcal{L}_{p_{1}}^{(a)}\left(A, B_{1}\right)\right)_{\theta, q}$ and let $T=\sum_{m=-\infty}^{\infty} T_{m}$ be any $J$-representation of $T$ with $\left(T_{m}\right) \subset \mathcal{L}_{p_{0}}^{(a)}\left(A, B_{0}\right) \cap \mathcal{L}_{p_{1}}^{(a)}\left(A, B_{1}\right)$. Since the constant in the triangle inequality for $\sigma_{p}$ is $2^{\frac{1}{p}} \max \left\{2^{\frac{1}{p}-1}, 1\right\}$ and $\left(2 \cdot 2^{1 / p} \max \left\{2^{1 / p-1}, 1\right\}\right)^{q}=2$, we derive from our previous estimates that

$$
\begin{aligned}
\sigma_{p}\left(T_{A,\left(B_{0}, B_{1}\right)_{\theta, 1}}\right) & \leq 2^{\frac{1}{q}}\left(\sum_{m=-\infty}^{\infty} \sigma_{p}\left(T_{m}\right)^{q}\right)^{\frac{1}{q}} \\
& \leq C\left(\sum_{m=-\infty}^{\infty}\left(\frac{J\left(2^{m}, T_{m}\right)}{2^{\theta m}}\right)^{q}\right)^{\frac{1}{q}} .
\end{aligned}
$$

This implies a).

A similar argument, but using Lemma 4.7/b and (2.10), proves b).

Remark 4.9. In fact, Lemma 4.7 is valid for any additive $s$ function $\mathbf{s}$ in the sense of Pietsch [18] and [19], and Theorem 4.8 works for operator ideals generated by any additive $s$-function.

\section{Injectivity and surjectivity}

Let $[\mathcal{J}, \tau]$ be a quasi-normed operator ideal. An operator $S \in \mathcal{L}(A, B)$ belongs to the surjective hull $\mathcal{J}^{\text {sur }}$ if there are a Banach space $E$ and an operator $T \in \mathcal{J}(E, B)$ so that $S\left(\mathcal{U}_{A}\right) \subseteq T\left(\mathcal{U}_{E}\right)$ where $\mathcal{U}_{A}$ and $\mathcal{U}_{E}$ stand for the closed unit balls of $A$ and $E$, respectively. The function

$$
\tau^{\text {sur }}(S)=\inf \left\{\tau(T): S\left(\mathcal{U}_{A}\right) \subseteq T\left(\mathcal{U}_{E}\right)\right\}
$$

defines a quasi-norm in $\mathcal{J}^{\text {sur }}$ and $\left[\mathcal{J}^{\text {sur }}, \tau^{\text {sur }}\right]$ turns out to be a quasinormed operator ideal (see [18]). It is clear that $c_{\tau^{\text {sur }}} \leq c_{\tau}$.

An operator $S \in \mathcal{L}(A, B)$ is said to belong to the injective hull $\mathcal{J}^{\text {inj }}$ if there are a Banach space $F$ and an operator $T \in \mathcal{J}(A, F)$ such that

$$
\|S x\|_{B} \leq\|T x\|_{F} \text { for all } x \in A \text {. }
$$

The quasi-norm on $\mathcal{J}^{\text {inj }}$ is given by

$$
\tau^{\operatorname{inj}}(S)=\inf \left\{\tau(T):\|S x\|_{B} \leq\|T x\|_{F}, x \in A\right\}
$$

and $\left[\mathcal{J}^{\text {inj }}, \tau^{\mathrm{inj}}\right]$ is a quasi-normed operator ideal (see [18]). Again $c_{\tau^{\mathrm{inj}}} \leq$ $c_{\tau}$.

If $[\mathcal{J}, \tau]=\left[\mathcal{J}^{\text {sur }}, \tau^{\text {sur }}\right]$ (resp. $[\mathcal{J}, \tau]=\left[\mathcal{J}^{\text {inj }}, \tau^{\text {inj }}\right]$ ), then the quasinormed operator ideal $\mathcal{J}$ is called surjective (resp. injective). Of course $[\mathcal{L},\|\cdot\|]$ satisfies these two conditions. Other examples are compact operators $[\mathcal{K},\|\cdot\|]$ and weakly compact operators $[\mathcal{W},\|\cdot\|]$. A normed operator ideal which is injective but fails to be surjective is the ideal $[\Pi, \pi]$ 
of all absolutely summing operators. Recall that $T \in \mathcal{L}(A, B)$ is absolutely summing if there is a constant $c>0$ such that for every finite set $\left\{a_{j}\right\}_{j=1}^{n} \subseteq A$

$$
\sum_{j=1}^{n}\left\|T a_{j}\right\|_{B} \leq c \sup \left\{\sum_{j=1}^{n}\left|f\left(a_{j}\right)\right|: f \in \mathcal{U}_{A^{*}}\right\} .
$$

We write $\pi(T)$ for the least constant $c$ for which the above inequality holds. We refer to [18] and [4] for more details on these notions.

Given two Banach spaces $E, F$, we denote by $E \oplus F$ the direct sum of $E$ and $F$, normed by $\|(x, y)\|=\max \left\{\|x\|_{E},\|y\|_{F}\right\}$.

We shall use some ideas developed in $[\mathbf{3}]$ to establish the following theorems.

Theorem 5.1. Let $\bar{B}=\left(B_{0}, B_{1}\right)$ be a regular Banach couple, let $B$ be an intermediate space with respect to $\bar{B}$ with $B_{0} \cap B_{1}$ dense in $B$ and let $\psi(t)$ be the $\psi$-function associated to $B$ and $\bar{B}$. If $A$ is any Banach space, $[\mathcal{J}, \tau]$ is a quasi-normed operator ideal and $q$ is defined by $\left(2 c_{\tau}\right)^{q}=2$, then

$$
\left(\mathcal{J}^{\text {sur }}\left(B_{0}, A\right), \mathcal{J}^{\text {sur }}\left(B_{1}, A\right)\right)_{\psi^{*}, q ; J} \hookrightarrow \mathcal{J}^{\text {sur }}(B, A) .
$$

Proof: Let $T \in \mathcal{J}^{\operatorname{sur}}\left(B_{0}, A\right) \cap \mathcal{J}^{\text {sur }}\left(B_{1}, A\right)$. Take any $\epsilon>0$, and let $E_{j}(j=0,1)$ be Banach spaces and $R_{j} \in \mathcal{J}\left(E_{j}, A\right)$ so that $T\left(\mathcal{U}_{B_{j}}\right) \subseteq$ $R_{j}\left(\mathcal{U}_{E_{j}}\right)$ and $\tau\left(R_{j}\right) \leq(1+\epsilon) \tau^{\mathrm{sur}}\left(T_{B_{j}, A}\right)$.

Given any $b \in \mathcal{U}_{B}$, we can find $b_{j} \in B_{j}$ such that $b=b_{0}+b_{1}$ and $\left\|b_{0}\right\|_{B_{0}}+t\left\|b_{1}\right\|_{B_{1}} \leq(1+\epsilon) \psi(t)$. Hence

$$
\mathcal{U}_{B} \subseteq(1+\epsilon) \psi(t) \mathcal{U}_{B_{0}}+(1+\epsilon) \frac{\psi(t)}{t} \mathcal{U}_{B_{1}} .
$$

Consider the Banach space $E_{0} \oplus E_{1}$ and the operator

$$
R(x, y)=(1+\epsilon) \psi(t) R_{0} x+(1+\epsilon) \frac{\psi(t)}{t} R_{1} y .
$$

Then $R \in \mathcal{J}\left(E_{0} \oplus E_{1}, A\right)$ and

$$
T\left(\mathcal{U}_{B}\right) \subseteq(1+\epsilon) \psi(t) R_{0}\left(\mathcal{U}_{E_{0}}\right)+(1+\epsilon) \frac{\psi(t)}{t} R_{1}\left(\mathcal{U}_{E_{1}}\right) \subseteq R\left(\mathcal{U}_{E_{0} \oplus E_{1}}\right) .
$$

Consequently,

$$
\begin{aligned}
\tau^{\mathrm{sur}}\left(T_{B, A}\right) & \leq \tau(R) \leq c_{\tau}(1+\epsilon)^{2} \psi(t)\left[\tau^{\mathrm{sur}}\left(T_{B_{0}, A}\right)+\frac{1}{t} \tau^{\mathrm{sur}}\left(T_{B_{1}, A}\right)\right] \\
& \leq 2 c_{\tau}(1+\epsilon)^{2} \frac{J\left(t^{-1}, T\right)}{\psi^{*}\left(t^{-1}\right)}
\end{aligned}
$$

Combining this estimate with [1, Lemma 3.10.2], the result follows. 
Since $[\mathcal{J}, \tau] \hookrightarrow\left[\mathcal{J}^{\text {sur }}, \tau^{\text {sur }}\right]$, we also have that

$$
\left(\mathcal{J}\left(B_{0}, A\right), \mathcal{J}\left(B_{1}, A\right)\right)_{\psi^{*}, q ; J} \hookrightarrow \mathcal{J}^{\operatorname{sur}}(B, A)
$$

This means that the assumption that $\bar{B}$ is quasi-linearizable in Theorem 3.8 and Corollary $3.9 / \mathrm{b}$ can be eliminated, provided we take $\left[\mathcal{J}^{\text {sur }}, \tau^{\text {sur }}\right]$ as the last operator space.

Writing down Theorem 5.1 for $B=\left(B_{0}, B_{1}\right)_{\theta, \infty}^{o}$ and $\mathcal{J}$ surjective we conclude:

Corollary 5.2. Let $\bar{B}=\left(B_{0}, B_{1}\right)$ be a regular Banach couple and let $A$ be a Banach space. If $[\mathcal{J}, \tau]$ is a surjective quasi-normed operator ideal, $\left(2 c_{\tau}\right)^{q}=2$ and $0<\theta<1$, then

$$
\left(\mathcal{J}\left(B_{0}, A\right), \mathcal{J}\left(B_{1}, A\right)\right)_{\theta, q} \hookrightarrow \mathcal{J}\left(\left(B_{0}, B_{1}\right)_{\theta, \infty}^{o}, A\right) .
$$

Next we show by means of an example that surjectivity is essential in the former result. We shall use the norm ideal $\Pi$ which is not surjective.

Example 5.3. Let $\Omega=c_{0} \oplus \ell_{1} \oplus \ell_{1}$ and let $\bar{A}=\left(A_{0}, A_{1}\right)$ be the Banach couple constructed by Garling and Montgomery-Smith in [9, Theorem 2]. Then $A_{j}$ is isometric to $\ell_{1}$; the projection $P: \Omega \longrightarrow c_{0}$ given by $P(x, y, z)=x$ belongs to $\mathcal{L}\left(\bar{A}_{\theta, \infty}^{o}, c_{0}\right)$ and the embedding $J: c_{0} \longrightarrow \Omega$ given by $J x=(x, 0,0)$ belongs to $\mathcal{L}\left(c_{0}, \bar{A}_{\theta, \infty}^{o}\right)$. Moreover, it is easy to check that $\bar{A}$ is regular.

Let now $R \in \mathcal{L}\left(c_{0}, \ell_{2}\right)$ be the operator defined by $R\left(\zeta_{n}\right)=\left(\zeta_{n} / n\right)$. A direct computation shows that $R \notin \Pi\left(c_{0}, \ell_{2}\right)$. Put $T=R P$. Since $A_{j}$ is isometric to $\ell_{1}$, Grothendieck's theorem implies that

$$
T \in \Pi\left(A_{0}, \ell_{2}\right) \cap \Pi\left(A_{1}, \ell_{2}\right) .
$$

However, $T$ does not belongs to $\Pi\left(\bar{A}_{\theta, \infty}^{o}, \ell_{2}\right)$ because $T J=R P J=R$ which is not absolutely summing.

We consider now the injective hull. 
Theorem 5.4. Let $\bar{B}=\left(B_{0}, B_{1}\right)$ be a Banach couple, let $B$ be an intermediate space with respect to $\bar{B}$ and let $\rho(t)$ be the $\rho$-function associated to $B$ and $\bar{B}$. Assume that $A$ is any Banach space, that $[\mathcal{J}, \tau]$ is a quasinormed operator ideal and let $q$ be defined by $\left(2 c_{\tau}\right)^{q}=2$. Then

$$
\left(\mathcal{J}^{\text {inj }}\left(A, B_{0}\right), \mathcal{J}^{\text {inj }}\left(A, B_{1}\right)\right)_{\rho, q ; J} \hookrightarrow \mathcal{J}^{\text {inj }}(A, B) .
$$

Proof: Let $T \in \mathcal{J}^{\text {inj }}\left(A, B_{0}\right) \cap \mathcal{J}^{\text {inj }}\left(A, B_{1}\right)$. Given any $\epsilon>0$ we can find Banach spaces $F_{j}(j=0,1)$ and operators $R_{j} \in \mathcal{J}\left(A, F_{j}\right)$ with $\|T x\|_{B_{j}} \leq\left\|R_{j} x\right\|_{F_{j}}$ for all $x \in A$, and $\tau\left(R_{j}\right) \leq(1+\epsilon) \tau^{\mathrm{inj}}\left(T_{A, B_{j}}\right)$. Given $t>0$, put $R x=\left(R_{0} x, t R_{1} x\right)$. Then $R \in \mathcal{J}\left(A, F_{0} \oplus F_{1}\right)$. It follows from

$$
\|T(x)\|_{B} \leq \frac{J(t, T x)}{\rho(t)} \leq \frac{1}{\rho(t)} \max \left\{\left\|R_{0} x\right\|_{F_{0}}, t\left\|R_{1} x\right\|_{F_{1}}\right\}=\frac{1}{\rho(t)}\|R x\|_{F_{0} \oplus F_{1}}
$$

that $T \in \mathcal{J}^{\text {inj }}(A, B)$ with

$$
\tau^{\mathrm{inj}}\left(T_{A, B}\right) \leq \frac{1}{\rho(t)} \tau(R) \leq \frac{c_{\tau}}{\rho(t)}\left(\tau\left(R_{0}\right)+t \tau\left(R_{1}\right)\right) \leq \frac{2 c_{\tau}(1+\epsilon)}{\rho(t)} J(t, T) .
$$

Now we conclude the result by appealing to [1, Lemma 3.10.2].

Since $[\mathcal{J}, \tau] \hookrightarrow\left[\mathcal{J}^{\text {inj }}, \tau^{\text {inj }}\right]$, Theorem 5.4 shows that we can eliminate the assumption of quasi-linearizability in Theorem 3.7 and Corollary 3.9 /a provided we take $\mathcal{J}^{\text {inj }}(A, B)$ as the last operator space. In particular, we have

Corollary 5.5. Let $\bar{B}=\left(B_{0}, B_{1}\right)$ be a Banach couple and let $A$ be a Banach space. If $[\mathcal{J}, \tau]$ is an injective quasi-normed operator ideal, $\left(2 c_{\tau}\right)^{q}=2$ and $0<\theta<1$, then

$$
\left(\mathcal{J}\left(A, B_{0}\right), \mathcal{J}\left(A, B_{1}\right)\right)_{\theta, q} \hookrightarrow \mathcal{J}\left(A,\left(B_{0}, B_{1}\right)_{\theta, 1}\right) .
$$

Working with the dual ideal $\left[\Pi^{d}, \pi^{d}\right]$ of absolutely summing operators, it is not hard to derive from Example 5.3 that injectivity is essential in Corollary 5.5.

Acknowledgement. We are grateful to Quanhua Xu for fruitful discussions and for drawing our attention to Lemma 4.4. We would also like to thank the referee for his comments.

\section{References}

[1] J. Bergh AND J. LÖFström, "Interpolation spaces. An introduction", Grundlehren der Mathematischen Wissenschaften 223, Springer-Verlag, Berlin, 1976. 
[2] F. Cobos, M. Cwikel and P. Matos, Best possible compactness result of Lions-Peetre type, Proc. Edinburgh Math. Soc. (2) (to appear).

[3] F. Cobos, A. Manzano and A. Martínez, Interpolation theory and measures related to operator ideals, Quart. J. Math. Oxford Ser. (2) 50(200) (1999), 401-416.

[4] J. Diestel, H. Jarchow and A. Tonge, "Absolutely summing operators", Cambridge Studies in Advanced Mathematics 43, Cambridge University Press, Cambridge, 1995.

[5] A. A. DMitriev, The interpolation of one-dimensional operators, Voronež. Gos. Univ. Trudy Naučn.-Issled. Inst. Mat. VGU (Vyp. 11 Sb. Statei Funkcional. Anal. i Prilozen.) 11 (1973), 31-43, (Russian).

[6] W. F. Donoghue, The interpolation of quadratic norms, Acta Math. 118 (1967), 251-270.

[7] D. E. Edmunds and D. D. Haroske, Embeddings in spaces of Lipschitz type, entropy and approximation numbers, and applications, J. Approx. Theory 104(2) (2000), 226-271.

[8] D. E. Edmunds And H. TRIEBEL, "Function spaces, entropy numbers, differential operators", Cambridge Tracts in Mathematics 120, Cambridge University Press, Cambridge, 1996.

[9] D. J. H. Garling and S. J. Montgomery-Smith, Complemented subspaces of spaces obtained by interpolation, J. London Math. Soc. (2) 44(3) (1991), 503-513.

[10] J. Gustavsson and J. Peetre, Interpolation of Orlicz spaces, Studia Math. 60(1) (1977), 33-59.

[11] O. KoubA, On the interpolation of injective or projective tensor products of Banach spaces, J. Funct. Anal. 96(1) (1991), 38-61.

[12] P. Nilsson, Reiteration theorems for real interpolation and approximation spaces, Ann. Mat. Pura Appl. (4) 132 (1982), 291-330.

[13] V. I. Ovchinnikov, Interpolation in symmetrically normed ideals of operators that act in different spaces, Funktsional. Anal. $i$ Prilozhen. 28(3) (1994), 80-82; translation in Funct. Anal. Appl. 28(3) (1994), 213-215.

[14] V. I. Ovchinnikov, Lions-Peetre construction for couples of operator spaces, Russian J. Math. Phys. 3(3) (1995), 407-410.

[15] J. Peetre, A theory of interpolation of normed spaces, Notas de Matemática 39, Instituto de Matemática Pura e Aplicada, Conselho Nacional de Pesquisas, Rio de Janeiro, 1968.

[16] J. Peetre, Zur Interpolation von Operatorenräumen, Arch. Math. (Basel) 21 (1970/71), 601-608. 
[17] J. Peetre, On the connection between the theory of interpolation spaces and approximation theory, in "Proceedings of the Conference on the Constructive Theory of Functions (Approximation Theory)" (Budapest, 1969), Akadémiai Kiadó, Budapest, 1972, pp. 351-363.

[18] A. Pietsch, "Operator ideals", North-Holland Mathematical Library 20, North-Holland Publishing Co., Amsterdam, 1980.

[19] A. Pietsch, "Eigenvalues and s-numbers", Cambridge Studies in Advanced Mathematics 13, Cambridge University Press, Cambridge, 1987.

[20] E. Pustylnik, Embedding functions and their role in interpolation theory, Abstr. Appl. Anal. 1(3) (1996), 305-325.

[21] H. TRIEBEL, "Interpolation theory, function spaces, differential operators", North-Holland Mathematical Library 18, North-Holland Publishing Co., Amsterdam, 1978.

Departamento de Análisis Matemático

Facultad de Matemáticas

Universidad Complutense de Madrid

28040 Madrid

Spain

E-mail address: cobos@eucmax.sim.ucm.es

E-mail address: Tesera_Signes@mat.ucm.es

Primera versió rebuda el 26 d'octubre de 1999, darrera versió rebuda el 2 de maig de 2000. 